Louisiana State University

LSU Digital Commons

$12-1-2010$

\title{
Application of a fast proton dose calculation algorithm to a thorax geometry
}

\author{
Pablo P. Yepes \\ Rice University \\ Travis Brannan \\ Rice University \\ Jessie Huang \\ Rice University \\ Dragan Mirkovic \\ University of Texas MD Anderson Cancer Center \\ Wayne D. Newhauser \\ University of Texas MD Anderson Cancer Center
}

See next page for additional authors

Follow this and additional works at: https://digitalcommons.Isu.edu/physics_astronomy_pubs

\section{Recommended Citation}

Yepes, P., Brannan, T., Huang, J., Mirkovic, D., Newhauser, W., Taddei, P., \& Titt, U. (2010). Application of a fast proton dose calculation algorithm to a thorax geometry. Radiation Measurements, 45 (10), 1367-1368. https://doi.org/10.1016/j.radmeas.2010.05.022

This Conference Proceeding is brought to you for free and open access by the Department of Physics \& Astronomy at LSU Digital Commons. It has been accepted for inclusion in Faculty Publications by an authorized administrator of LSU Digital Commons. For more information, please contact ir@lsu.edu. 


\section{Authors}

Pablo P. Yepes, Travis Brannan, Jessie Huang, Dragan Mirkovic, Wayne D. Newhauser, Phillip J. Taddei, and Uwe Titt 


\title{
Application of a fast proton dose calculation algorithm to a thorax geometry
}

\author{
Pablo P. Yepes ${ }^{a},{ }^{,}$, Travis Brannan ${ }^{a}$, Jessie Huang ${ }^{a}$, Dragan Mirkovic ${ }^{b}$, Wayne D. \\ Newhauser ${ }^{\mathrm{b}}$, Phillip J. Taddei ${ }^{\mathrm{b}}$, and Uwe Titt ${ }^{\mathrm{b}}$ \\ a Department of Physics and Astronomy, Rice University, MS 315, 6100 Main Street, Houston, \\ TX 77005, USA \\ b Department of Radiation Physics, Unit 1202, The University of Texas M. D. Anderson Cancer \\ Center, 1515 Holcombe Blvd., Houston, TX 77030, USA
}

\begin{abstract}
Treatment planning in proton therapy requires the calculation of absorbed dose distributions on beam shaping components and the patient anatomy. Analytical pencil-beam dose algorithms commonly used are not always accurate enough. The Monte Carlo approach is more accurate but extremely computationally intensive. The Fast Dose Calculator, a track-repeating algorithm, has been proposed as an alternative fast and accurate dose calculation. In this work FDC is applied to a proton therapy patient thoracic anatomy.
\end{abstract}

\section{Keywords}

Proton; Radiation; Therapy; Monte Carlo; Fast; Track-repeating; Lung; Thorax

\section{Introduction}

For some tumors proton therapy may reduce the risk of normal-tissue complications such as radiogenic second cancers. Such an advantage has led to a proliferation of proton cancer therapy centers worldwide. As of 2009, there are 30 operational hadron-therapy centers worldwide and 22 others are in various stages of construction or planning. Because of the sharp lateral and distal falloff of proton dose distributions, dosimetric and spatial accuracy in treatment planning is essential. Usually, commercial treatment plans calculate dose distributions with analytical pencil-beam dose algorithms (Schaffner et al., 1999), which do not always provide accurate results (Ciangaru et al., 2005). Analytical algorithms' inaccuracies are most important in inhomogeneous media like the thoracic and head-andneck anatomic sites. The Monte Carlo method provides superior accuracy (Titt et al., 2008; Koch et al., 2008), however it is very computationally intense. Hence its utilization for clinical treatment planning is challenging. The Fast Dose Calculator (FDC), a trackrepeating algorithm (Yepes et al., 2009a,b), has been shown to provide accurate and fast dose calculations. FDC reproduces Monte Carlo dose calculations within 2\%, while reducing computational times by more than two orders of magnitude. The objective of this study was to test the feasibility of applying FDC to the highly heterogeneous anatomy of the human thorax. Dose calculation improvements for that anatomic site might have an important impact in the accuracy of proton therapy treatment plans. The human thorax is a

(c) 2010 Elsevier Ltd. All rights reserved.

"Corresponding author. Tel.: p1 713348 5942; fax: p1 713348 5215. yepes@ rice.edu (P.P. Yepes). 
particularly challenging geometry due to the large inhomogeneities: high density areas, like bone, are contiguous to very low density media, like the lungs.

\section{Methods}

An irradiated patient, a range compensator and a collimating aperture were simulated in GEANT4 (Agostinelli et al., 2003; Allison et al., 2006) and FDC. The irradiated patient was represented as a voxelized phantom based on the $\mathrm{CT}$ images of the thoracic region of a typical patient who had previously been treated for lung cancer at The University of Texas M. D. Anderson Cancer Center. The phantom contained 6,064,305 voxels, each having dimensions of $1 \times 1 \times 2.5 \mathrm{~mm}^{3}$. Each voxel was assigned a material composition and density that corresponded to the Hounsfield unit value in the CT scan for that voxel, following the approach described elsewhere (Newhauser et al., 2008).

The field-defining collimating aperture (i.e., tumor-shaped block) and range compensator were also modeled. Only one of the fields utilized to treat the patient was implemented in the study. Moreover a $10 \mathrm{~cm}$ radius circular field with $140 \mathrm{MeV}$ fixed energy was utilized for simplicity. The FDC algorithm and the computational facilities were described in previous studies (Yepes et al., 2009a,b). For the current study a DICOM interface was implemented in FDC, which allows the program to read the patient anatomy data and field definition directly from the files generated by the clinical treatment planning system.

\section{Results}

Fig. 1 shows the dose distribution through the axial plane $(z=-17.5 \mathrm{~mm})$ for both GEANT4 and FDC. Good agreement was observed between the doses calculated by each code. A more comprehensive comparison was obtained by calculating the $\Gamma$ index of FDC relative to GEANT4 for each voxel (Low et al., 1998). A $\Gamma$ value below unity is considered satisfactory. The $\Gamma$ index results are presented in Fig. 2, as the complimentary cumulative distribution function such, that the ordinate represents the probability that $\Gamma$ will be greater than the value of the abscissa. Less than $0.1 \%$ of the voxels had $\Gamma$ values larger than unity. Thus, the dose distribution from FDC is in good agreement with the reference dose distribution from GEANT4. The time to process one history was $2.9 \mathrm{~s}$ and $0.9 \mathrm{~ms}$ for GEANT4 and FDC, respectively.

\section{Conclusions}

The dosimetric accuracy of FDC was tested in a particularly challenging anatomic geometry: the thorax. The acceptable level of agreement previously achieved for more homogeneous regions like the pelvis has been preserved. FDC shows gains up to a factor of one thousand with respect to GEANT4 for the patient phantom used in the study.

\section{References}

Agostinelli S, et al. GEANT4 a simulation toolkit. Nucl Instrum Methods Phys Res A. 2003; 506:250303.

Allison J, et al. Geant4 developments and applications. IEEE Trans Nucl Sci. 2006; 53:270-278.

Ciangaru G, Polf JC, Bues M, Smith AR. Benchmarking analytical calculations of proton doses in heterogeneous matter. Med Phys. 2005; 32:3511-3523. [PubMed: 16475750]

Koch N, Newhauser WD, Titt U, Gombos D, Coombes K, Starkschall G. Monte Carlo calculations and measurements of absorbed dose per monitor unit for the treatment of uveal melanoma with proton therapy. Phys Med Biol. 2008; 53:1581-1594. [PubMed: 18367789]

Low DA, Harms WB, Mutic S, Purdy JA. A technique for the quantitative evaluation of dose distributions. Med Phys. 1998; 25:65661. 
Newhauser WD, Zheng Y, Taddei PJ, Mirkovic D, Fontenot J, Giebeler A, Zhang R, Titt U, Mohan R. Monte Carlo proton radiation therapy planning calculations. Trans Am Nucl Soc. 2008; 99:63-64.

Schaffner B, Pedroni E, Lomax A. Dose calculation models for proton treatment planning using a dynamic beam delivery system: an attempt to include density heterogeneity effects in the analytical dose calculation. Phys Med Biol. 1999; 44:2741.

Titt U, Sahoo N, Ding X, Zheng Y, Newhauser WD, Zhu XR, Polf JC, Gillin MT, Mohan R. Assessment of the accuracy of an MCNPX-based Monte Carlo simulation model for predicting three-dimensional absorbed dose distributions. Phys Med Biol. 2008; 53:4455-4470. [PubMed: 18670050]

Yepes P, Randeniya S, Taddei P, Newhauser WD. A track repeating algorithm for fast Monte Carlo dose calculations of proton radiotherapy. Nucl Technol. 2009a; 168:334-337.

Yepes P, Randeniya S, Taddei P, Newhauser WD. Monte Carlo fast dose calculator for proton radiotherapy: application to a voxelized geometry representing a patient with prostate cancer. Phys Med Biol. 2009b; 54:N21-N28. (and references therein). [PubMed: 19075361] 

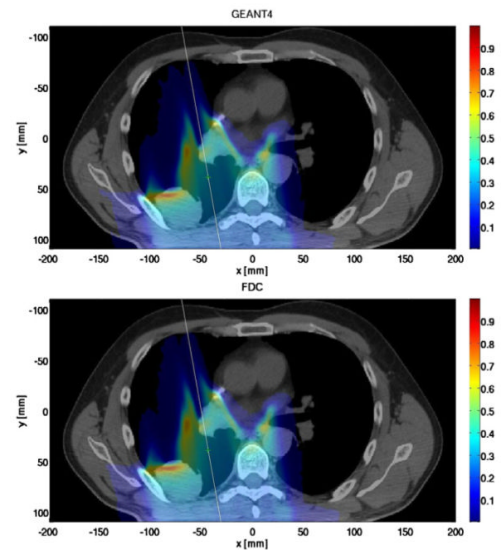

Fig. 1.

Dose distribution in a plane parallel to the circular mono-energetic $140 \mathrm{MeV}$ proton field calculated with GEANT4 (top) and FDC (bottom) for a patient being treated for lung cancer. 


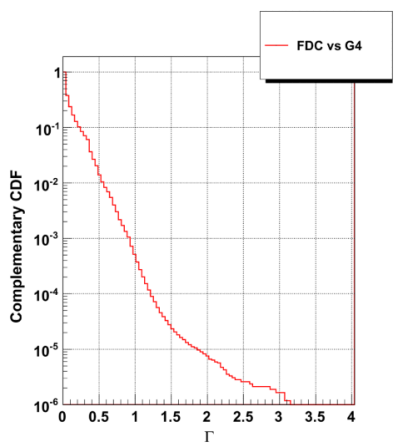

Fig. 2.

The complementary cumulative distribution function of the $\Gamma$ index for FDC using GEANT4 as reference for the phantom representing a patient being treated for lung cancer. 